\title{
Dynamics of Hydrophobic Core Phenylalanine Residues Probed by Solid-State Deuteron NMR
}

\author{
Liliya Vugmeyster \\ Dmitry Ostrovsky \\ Toni Villafranca \\ Wei Xu \\ College of William and Mary \\ Gina L. Hoatson \\ College of William and Mary \\ See next page for additional authors
}

Follow this and additional works at: https://scholarworks.wm.edu/aspubs

\section{Recommended Citation}

Vugmeyster, L., Ostrovsky, D., Villafranca, T., Sharp, J., Xu, W., Lipton, A. S., ... \& Vold, R. L. (2015).

Dynamics of hydrophobic core phenylalanine residues probed by solid-state deuteron NMR. The Journal of Physical Chemistry B, 119(47), 14892-14904. 


\section{Authors}

Liliya Vugmeyster, Dmitry Ostrovsky, Toni Villafranca, Wei Xu, Gina L. Hoatson, and Robert L. Vold 


\title{
Dynamics of Hydrophobic Core Phenylalanine Residues Probed by Solid-State Deuteron NMR
}

\author{
Liliya Vugmeyster, ${ }^{\dagger}{ }^{\dagger}$ Dmitry Ostrovsky, ${ }^{\dagger}$ Toni Villafranca, ${ }^{*}$ Janelle Sharp, ${ }^{*}$ Wei Xu, ${ }^{\S}$ Andrew S. Lipton, \\ Gina L. Hoatson, ${ }^{\S}$ and Robert L. Vold \\ ${ }^{\dagger}$ University of Colorado at Denver, Denver, Colorado 80204, United States \\ ${ }^{\ddagger}$ University of Alaska Anchorage, Anchorage, Alaska 99508, United States \\ ${ }^{\S}$ College of William and Mary, Williamsburg, Virginia 23187, United States \\ "Pacific Northwest National Laboratory, Richland, Washington 99354, United States
}

Supporting Information

\begin{abstract}
We conducted a detailed investigation of the dynamics of two phenylalanine side chains in the hydrophobic core of the villin headpiece subdomain protein (HP36) in the hydrated powder state over the 298-80 K temperature range. Our main tools were static deuteron NMR measurements of longitudinal relaxation and line shapes supplemented with computational modeling. The temperature dependence of the relaxation times reveals the presence of two main mechanisms that can be attributed to the ring-flips, dominating at high temperatures, and small-angle fluctuations, dominating at low temperatures. The relaxation is nonexponential at all temperatures with the extent of nonexponentiality increasing from higher to lower temperatures. This behavior suggests a distribution of conformers with unique values of activation energies. The central values of the activation energies for the ring-flipping

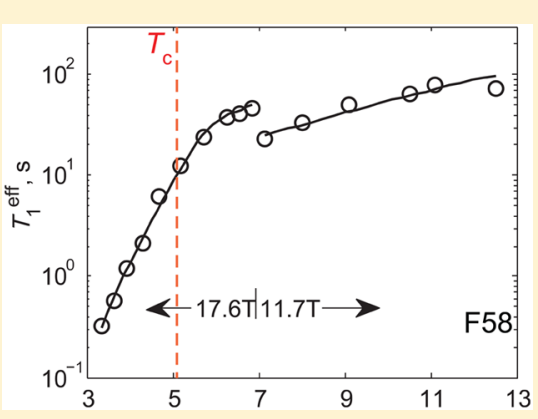
motions are among the smallest reported for aromatic residues in peptides and proteins and point to a very mobile hydrophobic core. The analysis of the widths of the distributions, in combination with the earlier results on the dynamics of flanking methyl groups (Vugmeyster et al. J. Phys. Chem. B 2013, 117, 6129-6137), suggests that the hydrophobic core undergoes slow concerted fluctuations. There is a pronounced effect of dehydration on the ring-flipping motions, which shifts the distribution toward more rigid conformers. The crossover temperature between the regions of dominance of the small-angle fluctuations and ring-flips shifts from $195 \mathrm{~K}$ in the hydrated protein to $278 \mathrm{~K}$ in the dry one. This result points to the role of solvent in softening the core and highlights aromatic residues as markers of the protein dynamical transitions.
\end{abstract}

\section{INTRODUCTION}

The hydrophobic core of a protein plays an essential role in a variety of biological functions and in defining protein stability and folding. ${ }^{1}$ It is known to be a very complex dynamical milieu with motions occurring on a variety of time scales from femtoseconds to seconds. ${ }^{2}$ The most common types of groups studied in regards to the hydrophobic environment in proteins are methyl groups. The dynamics of these groups are known to be sensitive not only to local fluctuations but also to the overall hydrophobic core environment. ${ }^{3-12}$ The assessment of the dynamics of aromatic side chains in a protein is relatively technically challenging, and much fewer studies are dedicated to these sites ${ }^{13-23}$ as compared to the investigations of protein methyl groups. Aromatic side chains comprise about $25 \%$ of the core volume on average and are often solvent-protected in the core by flanking methyl groups. The packing in the core is known to allow for a large extent of flexibility, even for the bulky aromatic groups of phenylalanines and tyrosines, which often participate in ring-flipping motions. The motions of these bulky groups likely require accommodations of the entire core, and thus their characterization is very important for the detailed assessment of the overall free energy landscape of the core. The notion of concerted core fluctuations or "breathing" has been suggested since the 1970s, and several works, such as those by Wagner et al., ${ }^{22,24}$ Hattori et al., ${ }^{18}$ and Otting et al., ${ }^{19}$ have characterized not only the activation enthalpy of ring-flipping motions but also the size of the cavity that has to be created in the core for ring-flipping motions to occur. A recent work by Kasinath et al. ${ }^{23}$ detected a dynamical transition at elevated temperatures, at which aromatic side chains undergo rotations consistent with continuous diffusion that do not require the formation of a cavity.

Recent advances in solution NMR spectroscopy and isotopic labeling strategies allow for a more precise characterization of ring-flipping motions and encompass a wider range of time scales. ${ }^{13,14}$ Solid state offers additional approaches for the characterization of motions in aromatic side chains on slow time scales due to the absence of overall molecular tumbling in the solid phase. In solution, overall molecular tumbling can often complicate the observation of motional modes on time

Received: September 23, 2015

Revised: November 1, 2015

Published: November 3, 2015 
scales close to or slower than those of the tumbling. Solid state also permits covering a much broader temperature range, thus, in principle, providing means for more thorough investigations of the energy landscape and its temperature dependence. Solidstate deuteron NMR techniques stand out as an important tool for the characterization of dynamics because deuteron NMR spectra and relaxation rates are usually dominated by one single-particle mechanism: the interaction of the nuclear electric quadrupole moment with the electric field gradient at the site of the nucleus. Available deuteron NMR techniques cover a very broad range of time scales, from pico- to milliseconds. ${ }^{25}$ The relaxation measurements of Zeeman and quadrupolar order coherences are most sensitive to fast motions in the pico- to nanosecond range, while line shape experiments report on slower time scales. Static deuteron NMR line shape and relaxation measurements have been used since the early 1980s to characterize the motions of aromatic side chains in a variety of systems from crystalline amino acids ${ }^{26,27}$ and amino acids bound to an enzyme ${ }^{17}$ to a multitude of polymer materials, ${ }^{28-30}$ to peptides, ${ }^{31-34}$ and, finally, to protein molecules. ${ }^{16,26,35}$ In particular, the work of Gall and Opella ${ }^{16}$ demonstrated that phenylalanine residues have a distribution of ring-flip rates. A recent work by $\mathrm{Li}$ et al. ${ }^{36}$ utilized a magic-angle spinning deuterium NMR approach to compare the dynamics of phenylalanine residues in microcrystalline and surface-bound states. While in the microcrystalline state, only a single rotamer is populated and the dynamics are dominated by ring-flipping motions, rotameric transitions are observed in the bound state.

In this work, we investigate the dynamics of two hydrophobic core phenylalanine residues in the chicken villin headpiece subdomain protein (HP36). We utilize static solid-state deuteron NMR and probe a very wide temperature range from room temperature to $80 \mathrm{~K}$. The challenge of obtaining sufficient sensitivity for the relaxation measurements for the full-width powder pattern spectra is partially overcome by the employment of the multiple-echo acquisition (QCPMG) technique. ${ }^{37}$ We then employ computational modeling to develop precise motional models consistent with the data over the entire temperature range. As a result, we characterize motional modes such as ring-flips and small-amplitude ring rotations and identify the crossover temperature separating the regions of dominance of these two modes.

The hydrophobic core of the HP36 protein is spanned by three helices with three phenylalanine side chains, F47, F51, and F58, forming the basis of the core (Figure 1). ${ }^{38-40}$ Several methyl groups flank the phenylalanine side chains, and we have previously conducted detailed investigations of their dynamics. $^{41-46}$ On the basis of the deuteron longitudinal relaxation rates in the $300-60 \mathrm{~K}$ temperature range, the dynamics of the core, as probed at all of the methyl sites, is characterized by a distribution of substates with different values of the activation energies for methyl rotations. ${ }^{42}$ We hypothesized that the origin of these substates may be caused by concerted variations in core packing. If this is the case, the dynamics of the bulky aromatic groups should also reflect the presence of multiple substates. We show the substates are indeed observed for both small-amplitude ring rotations and large-angle ring-flip modes.

\section{MATERIALS AND METHODS}

Sample Preparation and Conditions. The two core phenylalanines probed in this study are F51 and F58 (Figure 1). In each of the protein samples, the deuteron labels have been incorporated into only one of the two phenylalanine side

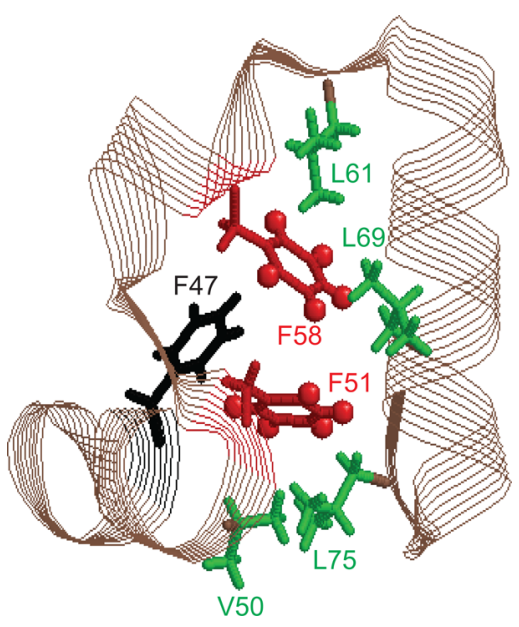

Figure 1. Ribbon diagram of the HP36 protein with the key hydrophobic core side chains labeled. F51 and F58, investigated in this work, are shown in red.

chains. Thus, the results are residue-specific. The labeling pattern (Figure 2) resulting from fluorenylmethyloxycarbonyl

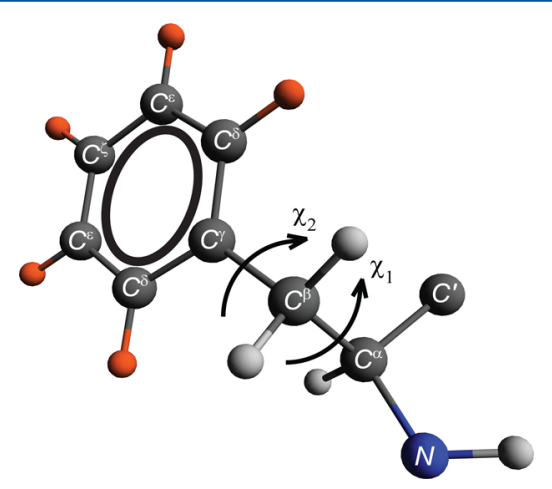

Figure 2. Phenylalanine side chain with deuteron labeling pattern marked in orange. Also shown are the torsional angles $\chi_{1}$ and $\chi_{2}$.

(FMOC)-phenylalanine-ring- $\mathrm{d}_{5}$ in the solid-state peptide synthesis corresponds to the deuterons incorporated in all five positions of the aromatic ring. The main sample conditions are hydrated powders with a water content of $40 \%$, which is a typical amount of water for protein powder samples. ${ }^{4-50} \mathrm{We}$ confirmed the refolding procedure by measuring the ${ }^{15} \mathrm{~N}$ backbone chemical shifts of the three core phenylalanines and additional leucine residues ${ }^{45,51}$ (Supporting Information SI1). An additional dry sample labeled at the F58 residue corresponds to lyophilized powder without the reintroduction of water.

The protein was prepared using solid-state peptide synthesis (performed by Thermofisher Scientific Co., Rockford, IL), and FMOC-phenylalanine-ring- $d_{5}$ was purchased from Cambridge Isotopes Laboratories (Andover, MA). The peptides were purified by reversed-phase HPLC, and identity and purity were confirmed by mass spectroscopy and reversed-phase HPLC. Lyophilized powders were dissolved in water, and the $\mathrm{pH}$ was adjusted to about 6 using $\mathrm{NaOH} / \mathrm{HCl}$. A hydrated state was achieved by exposing lyophilized powder to water vapor in a sealed chamber at $25{ }^{\circ} \mathrm{C}$ until the water content reached saturating levels corresponding to about $40 \%$ by weight. The samples, in amounts of $20-25 \mathrm{mg}$, were packed in $5 \mathrm{~mm}$ NMR 
tubes (cut to $21 \mathrm{~mm}$ length) using Teflon tape to center the sample volume in the coil.

Deuteron Solid-State NMR Spectroscopy. Experiments were performed at the 11.7 and $17.6 \mathrm{~T}$ magnetic fields. The 17.6 T WB750 Bruker spectrometer was equipped with AVANCE I electronics and a static probe operating in the range of temperatures between 145 and $380 \mathrm{~K}$. The $11.7 \mathrm{~T} \mathrm{WB}$ Varian Unity Plus spectrometer was equipped with a static cryogenic helium-cooled probe ${ }^{52}$ for temperatures below 150 K. $T_{1 Z}$ (Zeeman) measurements under static conditions were performed by the saturation recovery sequence. Because of the low sensitivity of the protein samples with dilute single-residue labels, an enhancement of the signal is necessary for the collection of accurate relaxation data. We made use of the multiple-echo acquisition scheme (QCPMG), ${ }^{37}$ which is a static analogue of magic-angle spinning. The scheme breaks a static powder pattern into a series of spikelets that follows the line shape (Figure 3).

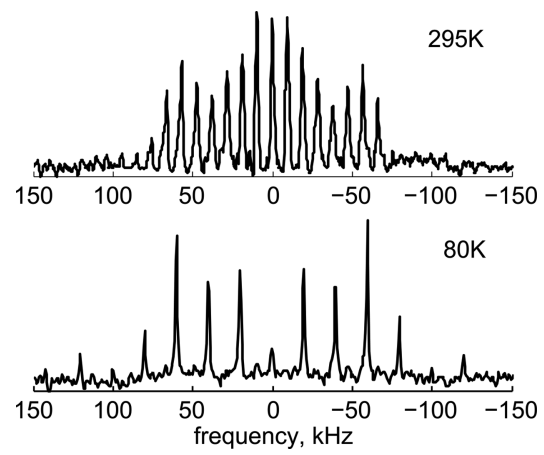

Figure 3. Experimental ${ }^{2} \mathrm{H}$ QCPMG spectra for the hydrated HP36 protein labeled at the F58-ring- $d_{5}$ site; data are shown for 295 and $80 \mathrm{~K}$.

Fifteen echoes were collected with $104 \mu$ s pulse spacing on the $17.6 \mathrm{~T}$ spectrometer and $50 \mu \mathrm{s}$ on the $11.7 \mathrm{~T}$ spectrometer, corresponding to QCPMG spikelets (sidebands) spaced at 10 and $20 \mathrm{kHz}$ intervals, respectively. The number of echoes was optimized to avoid sample heating. The durations of $90^{\circ}$ pulses were between 2.0 and $2.5 \mu \mathrm{s}$. Six to nine relaxation delays were collected. The number of scans varied greatly between 32 and 4096 depending on the signal-to-noise ratio in each sample and at each temperature as well as the precision of the data needed to define the nonexponential decays.

Room-temperature line shape measurements with the quadrupolar echo acquisition scheme were performed at 17.6 $\mathrm{T}$ with an eight-step phase cycle and a delay of $31 \mu$ s between $90^{\circ}$ pulses. The number of scans varied between $40 \times 1024$ and $250 \times 1024$, which was determined by the amount of the sample and whether it was in the dry or hydrated state. The delay between the scans was set to at least 2.5 times the effective relaxation time at each temperature. We confirmed that this is sufficient to obtain nondistorted line shapes. For the echo-delay dependence of line shapes, echo delays between 21.5 and $56.5 \mu$ s were employed. For the line shape measurement of FMOC-phenylalanine at $373 \mathrm{~K}$, the interscan delay was set to $120 \mathrm{~s}$ and the number of scans to 2048 . The spectra were processed with $1 \mathrm{kHz}$ exponential line broadening.

On the 17.6 $\mathrm{T}$ spectrometer, temperature calibration was done by recording static lead nitrate line shapes ${ }^{53}$ and using the freezing point of $\mathrm{D}_{2} \mathrm{O}, 3.8{ }^{\circ} \mathrm{C}$, as the fixed point for the calibration. The cryogenic probe on the $11.7 \mathrm{~T}$ spectrometer had a temperature sensor in immediate proximity to the sample area. Its reading was taken as the sample temperature.

Simulations. I. Relaxation. The spin-lattice relaxation rate $T_{1}$ is given $b^{7,54}$

$$
\frac{1}{T_{1}}=\frac{C_{\mathrm{q}}^{2}}{3}\left(J_{1}\left(\omega_{0}\right)+4 J_{2}\left(2 \omega_{0}\right)\right)
$$

where $\omega_{0}$ is the Larmor frequency, $J_{1}$ and $J_{2}$ are spectral density functions, and $C_{\mathrm{q}}$ refers to the quadrupole coupling constant (units are $\mathrm{Hz}$ ) in the absence of motion. $J_{1}$ and $J_{2}$ are dependent on the time scales and types of underlying motional processes as well as on the crystallite orientations. Spectral density functions can be obtained analytically for several simple models of motion. ${ }^{55}$ However, a description of the processes with multiple motional modes and/or multiple conformers usually requires computer simulations.

The model consistent with the relaxation data over the entire temperature range involves two modes of motions with rotations around the $\chi_{2}$ dihedral angle of the phenylalanine side chain. We describe these rotational modes in the so-called strong collision limit, such that they correspond to jumps between four sites, schematically represented in Figure 4A. The large-angle flips occur between sites $1-3$ and $1-4$ with an equal probability; this also holds for the $2-3$ and $2-4$ pairs. The small-angle jumps with the amplitude $\alpha$ occur between sites 12 and 3-4. The change in the $\chi_{2}$ angle for transitions $1-3$ and $2-4$ is exactly $180^{\circ}$ due to the symmetry of the ring.

The rotations around the $\chi_{2}$ angle do not involve the $\zeta$ (para) deuteron. Thus, only the $\mathrm{D}^{\delta}$ and $\mathrm{D}^{\varepsilon}$ deuterons participate in the jumps. We assume that the principal axis system of the quadrupolar tensor for each deuteron is aligned along the position of the $\mathrm{C}-\mathrm{D}$ bond. To simulate the jumps between the four sites, one has to define the angle between the $\mathrm{C}-\mathrm{D}$ bond and $C^{\beta}-C^{\gamma}$ axis as well as the angles of rotation around the $C^{\beta}-$ $\mathrm{C}^{\gamma}$ axis for each of the sites. The angle between the $\mathrm{C}-\mathrm{D}$ bond and $C^{\beta}-C^{\gamma}$ axis remains the same for all four exchange sites and, assuming an ideal ring geometry, equals $60^{\circ}$ for the $C^{\varepsilon}-D$ bonds and $120^{\circ}$ for the $\mathrm{C}^{\delta}-\mathrm{D}$ bonds. To specify the angle of rotation around the $\mathrm{C}^{\beta}-\mathrm{C}^{\gamma}$ axis (the $\chi_{2}$ rotation), only one parameter is required because the rotation angles between sites $1-2$ and 3-4 are equal and the transitions $1-3$ and 2-4 are exactly $180^{\circ}$. For example, if we model small-angle jumps by the amplitude of $\alpha$, this is the only parameter that needs to be specified.

The rate constants of transitions between the four positions are specified in the transition rate matrix:

$$
\left(\begin{array}{cccc}
-k^{\text {small }}-k^{\text {large }} & k^{\text {small }} & k^{\text {large }} / 2 & k^{\text {large }} / 2 \\
k^{\text {small }} & -k^{\text {small }}-k^{\text {large }} & k^{\text {large }} / 2 & k^{\text {large }} / 2 \\
k^{\text {large }} / 2 & k^{\text {large }} / 2 & -k^{\text {small }}-k^{\text {large }} & k^{\text {small }} \\
k^{\text {large }} / 2 & k^{\text {large }} / 2 & k^{\text {small }} & -k^{\text {small }}-k^{\text {large }}
\end{array}\right)
$$

where $k^{\text {small }}$ is the small-angle jump rate and $k^{\text {large }} / 2$ is the rate for large-angle jumps. For example, if small-angle jumps have the amplitude of $5^{\circ}$, then $k^{\text {large }} / 2$ corresponds to the rate of jump from $0^{\circ}$ to either $175^{\circ}$ or $180^{\circ}$. The diagonal elements are chosen to satisfy the transition rate matrix conditions.

It is possible to derive analytical expressions for the correlation functions determining the spectral densities $J_{1}$ and $J_{2}$ of eq 1 for this model. The correlation functions of the 

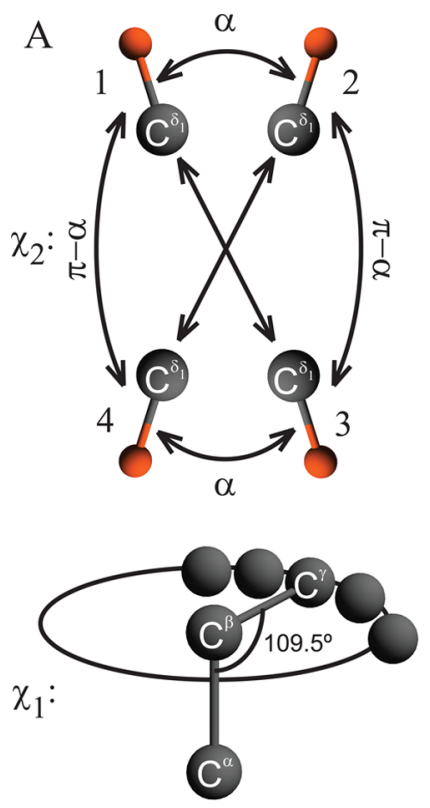

$\mathrm{B}$

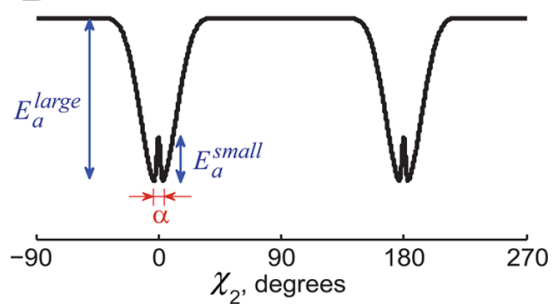

Figure 4. (A) Motional model for the fluctuations of the phenylalanine side chains. The motions around the $\chi_{2}$ dihedral angle consist of largeangle ring-flips and small-angle fluctuations, parametrized by angle $\alpha$. The diagram displays sites' connectivities according to the strong collision model, illustrated for one of the $\mathrm{C}^{\delta}-\mathrm{D}$ bonds. The motion around the $\chi_{1}$ dihedral angle is modeled via the restricted diffusion of the $\mathrm{C}^{\beta}-\mathrm{C}^{\gamma}$ axis along an arc and is approximated by small nearest neighbor jumps. (B) Potential corresponding to the model of motions around the $\chi_{2}$ angle, in which $E_{\mathrm{a}}^{\text {large }}$ and $E_{\mathrm{a}}^{\text {small }}$ are the activation energies for the large-angle ring-flips and small-angle fluctuations, respectively.

fluctuations around the phenyl axis are defined by $C_{p, q}(t)=$ $\left\langle\mathrm{e}^{-i p \gamma_{2}(t)} \mathrm{e}^{i q \gamma_{2}(0)}\right\rangle$, where \langle\rangle stands for the ensemble average and $p$ and $q$ denote the components of Wigner matrices responsible for the transformations between the laboratory and deuteron principal axis systems. Note that with the transitions $1-4$ and 2-3 taken into account, the correlation function cannot be decomposed into the product of independent terms responsible for small- and large-angle fluctuations. For example, the term $C_{1,1}(t)$ has the form:

$$
C_{1,1}(t)=C_{-1,-1}(t)=S^{2} \mathrm{e}^{-2 k^{\text {large }} t}+\left(1-S^{2}\right) \mathrm{e}^{-\left(k^{\text {large }}+2 k^{\text {small }}\right) t}
$$

in which $S=\cos (\alpha / 2)$ has the meaning of the order parameter for small-angle fluctuations. In the limit of time scale separation in which $k^{\text {large }} \ll k^{\text {small }}$, the two motional modes become decoupled. The full analytical treatment of the model is presented in Supporting Information SI2.

The full model also includes a distribution of conformers distinguished by their values of the activation energies for both large- $E_{\mathrm{a}}^{\text {large }}$ and small-angle jumps $E_{\mathrm{a}}^{\text {small. }}$. The distribution arises due to heterogeneity of the free energy landscape of the hydrophobic core. A more detailed description of the distribution of the activation energies can be achieved by introducing a coupling term between the degrees of freedom describing the motion of the nuclei of interest and their changing molecular environment. As an example, a recently developed extension (MOMD) to slowly relaxing local structure (SRLS) approach may be considered as a possible framework for such a coupling. ${ }^{56,57}$ The assumption of temperature independence of the distribution of the activation energies worked very well for our previous studies of methyl groups over the wide temperature range of $300-90 \mathrm{~K},{ }^{43}$ suggesting that the conformational substates giving rise to the distribution do not change their structures substantially over the entire temperature range. As we will see in the Results and Discussion, the assumption of temperature independence is also adequate for the description of the data on the phenylalanine residues. We also note that for a phenomenological application of the Arrhenius law, the linear term in the dependence of activation energy on temperature is equivalent to the rescaling of the pre-exponential factor and is, therefore, undetectable.

The temperature dependence of the rate constants for largeand small-angle jumps in each conformer is taken to be Arrhenius: $k\left(E_{\mathrm{a}}^{\text {large }}, T\right)=k_{0}^{\text {large }} \mathrm{e}^{-E_{\mathrm{a}}^{\text {large }} / T}$ and $k\left(E_{\mathrm{a}}^{\text {small }}, T\right)=$ $k_{0}^{\text {small }} \mathrm{e}^{-E_{a}^{\text {sall }} / T}$. The values of $k_{0}^{\text {large }}$ and $k_{0}^{\text {small }}$ were assumed to be the same for all conformers.

As magnetization buildup curves depend on the rate constants, the distributions in the activation energies become the source of the nonexponentiality of the magnetization buildup. For the case of a continuous distribution of conformers, the overall observable magnetization $M(t)$ is defined by

$$
M(t)=\iint m\left(E_{\mathrm{a}}^{\text {large }}, E_{\mathrm{a}}^{\text {small }}, t\right) \mathrm{d} E_{\mathrm{a}}^{\text {large }} \mathrm{d} E_{\mathrm{a}}^{\text {small }}
$$

in which $m\left(E_{\mathrm{a}}^{\text {large }}, E_{\mathrm{a}}^{\text {small }}, t\right)$ is the magnetization density. The magnetization density is proportional to the distribution functions for the activation energies. ${ }^{43}$ A simple Gaussian form of the distribution was used for the large-angle jumps with the central values denoted by $\left\langle E_{\mathrm{a}}^{\text {large }}\right\rangle$ and the width of $\sigma_{\text {large }}$, but an asymmetric gamma distribution was more appropriate for the small-angle jumps with the central value of $\left\langle E_{a}^{\text {small }}\right\rangle$ and the width of $\sigma_{\text {small }}$. They are related to the more conventional shape and rate parameters of the gamma distribution by $\left(\left\langle E_{\mathrm{a}}^{\text {small }}\right\rangle / \sigma_{\text {small }}\right)^{2}$ and $\left\langle E_{\mathrm{a}}^{\text {small }}\right\rangle / \sigma_{\text {small }}^{2}$, respectively. The longitudinal relaxation rate depends on the activation energies through the jump rate constants $k^{\text {small }}$ and $k^{\text {large }} / 2$.

All simulations were performed using the EXPRESS program, ${ }^{58}$ which allows for the incorporation of the QCPMG acquisition scheme. The values of the quadrupolar coupling constant $C_{\mathrm{q}}$ and asymmetry parameter $\eta$ of the tensor were chosen on the basis of the spectral data at the high and low temperature limits, which were consistent with the values of $C_{\mathrm{q}}=180 \mathrm{kHz}$ and $\eta=0$. The continuous distribution was approximated by 21 conformers in the $E_{\mathrm{a}}^{\text {large }}$ dimension and 11 conformers in the $E_{\mathrm{a}}^{\text {small }}$ dimension, analogous to the manner described previously for the methyl groups. The obtained $M(t)$ profiles were fitted by the stretch-exponential function defined in eq 5 to yield simulated values of $T_{1}^{\text {eff }}$ and $\beta$. Thus, a library of simulated parameters $T_{1}^{\text {eff }}$ and $\beta$ was created as a function of the small- and large-angle jump rates to fit the experimental data. The fitting was performed using $\chi^{2}$ minimization. 
II. Line Shapes. An additional motional mode was required to simulate the line shape data. This mode involves motions in the plane perpendicular to the $\pi$-flips axis and corresponds to fluctuations of the $\chi_{1}$ dihedral angle. The fluctuations were modeled as a restricted diffusion of the $C^{\beta}-C^{\gamma}$ axis on an arc, with the arc length as a fitting parameter that signifies the extent of variations in the $\chi_{1}$ angle. We thus approximate the actual potential restricting the dihedral angle fluctuations by flat potential (inside the arc) with rigid boundaries.

In EXPRESS, the introduction of this additional mode requires a two-frame simulation setup, in which the rotations around the $\chi_{2}$ angle are input in the first frame, which is nested inside the second frame defining the rotations around the $\chi_{1}$ angle. This embedding is parametrized by two angles, one of which is the angle between the $\mathrm{C}^{\alpha}-\mathrm{C}^{\beta}$ and $\mathrm{C}^{\beta}-\mathrm{C}^{\gamma}$ bonds and is defined by the tetrahedral geometry (fixed at $70.5^{\circ}$ ). The second angle defining the variations of $\chi_{1}$ is specified by the azimuthal angle of rotations of the $\mathrm{C}^{\beta}-\mathrm{C}^{\gamma}$ axis around the $\mathrm{C}^{\alpha}-$ $\mathrm{C}^{\beta}$ axis. In this case, the average $\chi_{2}$ angle has to be specified and we took it as $90^{\circ}$, which represents the most dominant rotameric configurations ${ }^{59}$ and is also consistent with what is found from the structural coordinates $\left(1 \mathrm{YRF}^{40}\right)$. The diffusion motion along the restricted arc was modeled by $5^{\circ}$ steps. EXPRESS uses the following convention: $k=1 / \tau_{\mathrm{c}}$. Within this notation, the diffusion coefficient in $\mathrm{rad}^{2} / \mathrm{s}$ is given by $D=$ $k(5 \pi / 180)^{2}$. The extent of variations in $\chi_{1}$ and the diffusion constant were chosen by visual inspection of an array of simulated spectra. The array consisted of variations from $10^{\circ}$ to $60^{\circ}$ in $5^{\circ}$ steps and variations in the diffusion constant from 80 to $1.5 \times 10^{3}$ in nine steps on a logarithmic scale. Simulated line shapes were processed with $1 \mathrm{kHz}$ exponential line broadening to match the experimental data processing and an additional 1.5 $\mathrm{kHz}$ Gaussian broadening to approximate the effect of homonuclear dipolar coupling on the line width. ${ }^{60}$

When included in the relaxation simulations, the correction due to this mode is on the order of $1 \%$. We also note that the motions around the $\chi_{1}$ angle include contributions from all ring positions, including the $\mathrm{D}^{\zeta}$ position. However, experimental line shapes at $295 \mathrm{~K}$ were collected with the relaxation delay on the order of $0.7-1.8$ (2.5 times the apparent central $T_{1}$ values, as detailed in Deuteron Solid-State NMR Spectroscopy), which saturates all signals from the $\mathrm{D}^{\zeta}$ deuterons and eliminates their contribution.

\section{RESULTS AND DISCUSSION}

Longitudinal Relaxation Measurements over the 300-80 K Temperature Range. The main relaxation mechanism in phenylalanine rings is usually considered to be the symmetrical $\pi$-flips of the ring around the $C^{\beta}-C^{\gamma}$ axis (Figure 4). We will see that our quantitative data over a very broad temperature range unveil many fine features of the landscape that complement the traditional view of phenylalanine ring-flips.

I. Nonexponentiality of Magnetization Buildup Curves Reports on the Presence of Multiple Conformational Substates. The saturation recovery buildup curves (Figure 5) reveal nonexponential behavior. The nonexponentiality of the magnetization buildup curves is evident starting at room temperature, and its extent gradually increases at lower temperatures. For consistency, all data shown correspond to the spikelets at $\pm 60 \mathrm{kHz}$, which are majors singularities at all temperatures except for the highest temperature of $295 \mathrm{~K}$ (Figure 3). There is no anisotropy in the data within the

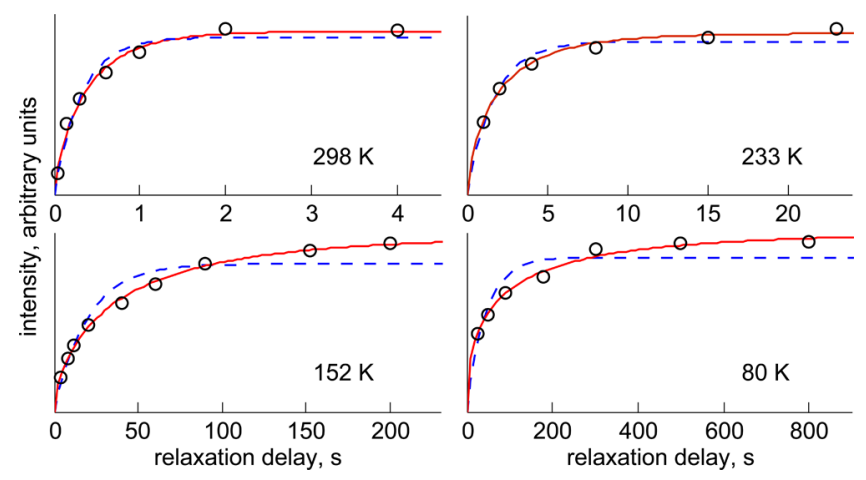

Figure 5. Longitudinal magnetization recovery curves at different temperatures are shown for the F58-ring- $\mathrm{d}_{5}$ site in the hydrated state. Signal intensities (in arbitrary units) are plotted as a function of the relaxation delay. The fits of the experimental data $(O)$ to the monoexponential function are shown as blue dashed lines, and the fits to the stretched exponential function are shown as red solid lines.

precision of the measurements. The data above $145 \mathrm{~K}$ were collected at $17.6 \mathrm{~T}$, and the data below $145 \mathrm{~K}$ were collected on a $11.6 \mathrm{~T}$ spectrometer equipped with a cryogenic helium-cooled probe. $^{52}$

Analogous to glassy systems, the nonexponential buildup curves can be fitted to a stretched-exponential function of the form: ${ }^{61-63}$

$$
I(t)=I(\infty)\left(1-\mathrm{e}^{-\left(t / T_{1}^{\mathrm{eff}}\right)^{\beta}}\right)
$$

in which $I(t)$ is the signal intensity, $T_{1}^{\text {eff }}$ is the effective relaxation time, and $\beta$ is the parameter that reflects the degree of nonexponentiality, $0<\beta \leq 1$. $\beta$ less than 1 corresponds to nonexponential behavior. In the absence of conformational exchange, this function can be viewed as an integral of ordinary monoexponential relaxation functions over a distribution of the relaxation rates. ${ }^{64}$

We have previously observed the nonexponentiality of the longitudinal relaxation for the hydrophobic core methyl groups. The relaxation data for these groups over the $300-60 \mathrm{~K}$ temperature range were fitted using a model consisting of a distribution of conformers distinguished by different values of activation energies for methyl three-site jumps. The widths of the distribution were on the order of $1.5 \mathrm{~kJ} / \mathrm{mol}$ for all four of the methyl residues probed. The existence of such distributions reflected multiple substates in the free energy landscape of the core, and we hypothesized that these substates represent concerted core repacking. The observation of nonexponentiality in the relaxation for the core phenylalanine ring deuterons suggests the presence of multiple substates for these residues, which can likely be modeled using a distribution of activation energies for ring rotations. Combined with the methyl data, the presence of multiple substates for the bulky aromatic side chains strongly supports the hypothesis of concerted core repacking.

II. Temperature Dependence of the Relaxation Profiles Indicates the Presence of Two Mechanisms. Plotting the values of the effective relaxation times $T_{1}^{\text {eff }}$ and $\beta$ as a function of inverse temperature (Figure 6) reveals an interesting pattern: the values of $\ln T_{1}^{\text {eff }}$ increase in a roughly linear fashion between room temperature and $170 \mathrm{~K}$ and start to level out below about $165 \mathrm{~K}$; the values of $\beta$ decrease with a decrease in temperature. This pattern of the temperature dependence of relaxation times suggests two different mechanisms: one with strong temper- 

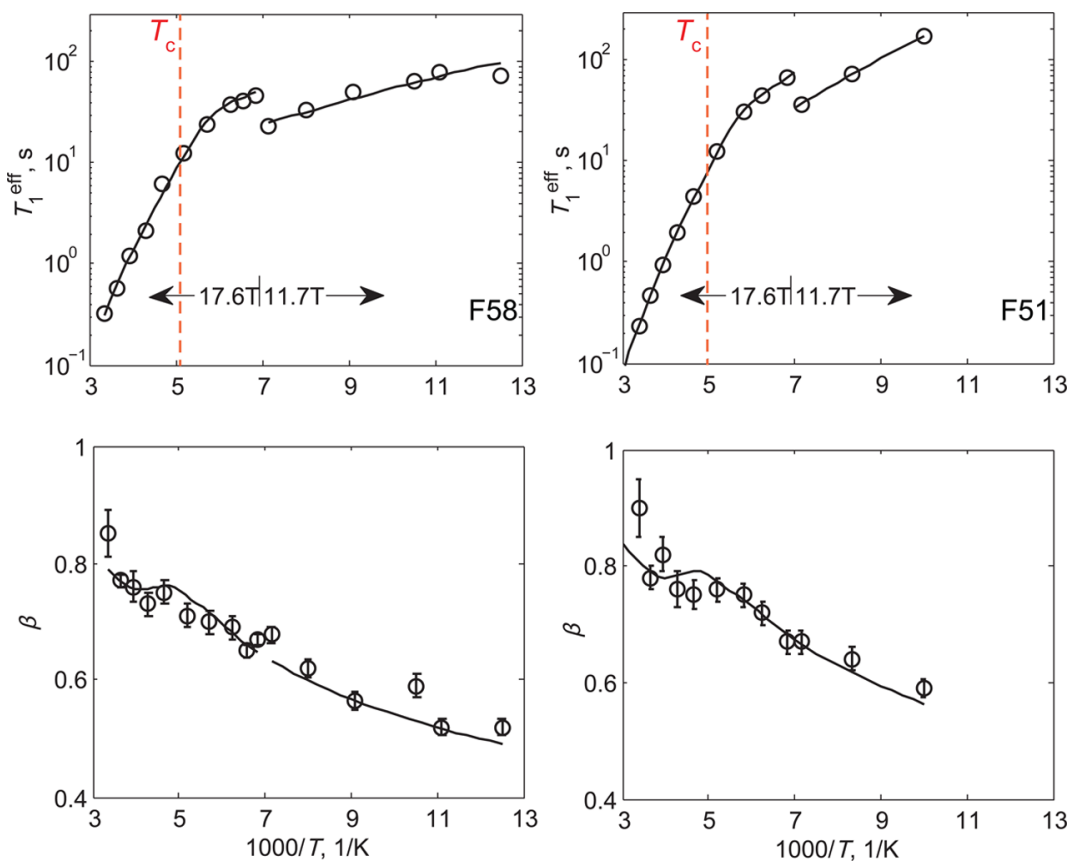

Figure 6. Plots of $T_{1}^{\text {eff }}$ versus $1000 / T$ on the semilog scale and $\beta$ versus $1000 / T$ for hydrated samples with perdeuterated rings of F51 and F58. Experimental data $(\mathrm{O})$ are fit to the model (solid lines), which involves large- and small-angle fluctuations around the $\chi_{2}$ angle, as described in the text and in Figure 3. The small-angle amplitude $\alpha=5^{\circ}$. The errors in $T_{1}^{\text {eff }}$ are within the size of the symbol. The red dotted vertical lines indicate the crossover temperature $T_{c}$.

ature dependence that dominates at high temperatures and another one with much weaker temperature dependence that is dominant at low temperatures. The likely dominant mechanism at high temperatures, consistent with the steep temperature dependence of relaxation times, is the large-angle fluctuations of the ring, which are essentially the $\pi$-flips. The relaxation mechanism with weak temperature dependence likely corresponds to the small-angle fluctuations of phenyl rings, which are expected to be dominant at low temperatures when the $\pi$ flips are suppressed due to their high activation energy barriers.

The decrease in the values of $\beta$ upon lowering the temperature indicates that for both types of mechanisms the distribution in rate constants becomes wider as the temperature is lowered. We remind that the distribution in the rate constants is responsible for the nonexponential behavior and causes $\beta$ to deviate from unity. As both processes appear thermally activated based on the temperature-dependent pattern of $T_{1}^{\text {eff }}$, the observed temperature dependence of $\beta$ suggests distributions in the values of the activation energies for both mechanisms. This will be discussed in more detail in the following section.

III. Fitting the Relaxation Profiles to the Two-Mode Motional Model. We model both large- and small-angle fluctuations as changes in the $\chi_{2}$ torsional angle (Figure 4). The symmetry of the ring implies that the crude picture of the phenylalanine ring potential should contain two minima of the $\chi_{2}$ angle $180^{\circ}$ apart, that is, represent a double well potential. The large-angle fluctuations represent transitions between the wells, and the small-angle fluctuations represent intrawell motions. However, the activation character of both types of fluctuations observed in the experiment means that the shape of the potential governing the ring dynamics is more complex than the traditional two minimum double well shape, as illustrated in Figure 4B. Inside each rather deep well, the potential displays an additional more shallow double well structure. Such a potential is a cause of the two different rate constants, $k^{\text {small }}$ and $k^{\text {large }}$.

The motion in this potential can, in principle, follow two different scenarios: (i) The first is the restricted diffusion case, in which the large-angle mode is coupled to the small-angle mode as described by Kramers' escape theory. ${ }^{65}$ In this limit, both large- and small-angle fluctuations are described by a single diffusion constant. (ii) The large-angle fluctuations occur in the strong collision limit, and there are two independent rate constants of large- and small-angle fluctuations. The strong collision limit implies that large-angle jumps can occur from any angle position inside the first well into any angle position inside the second well.

The first scenario puts strong restrictions on the possible values of the diffusion constant, because one needs to accommodate the widely different activation energies of the two motions and describe the experimentally observed crossover point between the dominance of the two regimes. In fact, we found that this scenario is incompatible with the experimental data and therefore concluded that the strong collision limit is most appropriate. Hattori et al. ${ }^{18}$ and Wagner ${ }^{24}$ also suggested that large-angle ring-flips occur in the strong collision limit. Their analysis was based on the comparison of the calculated and experimental volumes of the cavity formed in the core for the ring-flipping motion to take place. We have previously shown that the leveling of relaxation times at low temperatures in methyl groups can be explained by small-angle intrawell fluctuations in the restricted diffusion limit. ${ }^{66}$ The results of this work generalize this statement to either diffusion or strong collision limits.

We model the small-angle fluctuations as jumps between two discrete sites, and the strong collision limit for the large-angle fluctuations implies the following jump pattern: $\alpha \Leftrightarrow \pi, 0 \Leftrightarrow \pi$, $\alpha+\pi \Leftrightarrow 0, \alpha+\pi \Leftrightarrow \alpha$, where $\alpha$ is the angle of small amplitude 
Table 1. Fitted Parameters Characterizing the Large- $\left(175^{\circ}\right.$ and $\left.180^{\circ}\right)$ and Small-Angle $\left(5^{\circ}\right)$ Fluctuations in the Perdeuterated Phenylalanine Rings of the HP36 Protein in the Hydrated and Dry Powder States

$\begin{array}{ccccccc}\text { residue } / \text { conditions } & \ln k_{0}^{\text {large }}, \mathrm{s}^{-1} & \left\langle E_{\mathrm{a}}^{\text {large }}\right\rangle, \mathrm{kJ} / \mathrm{mol} & \sigma_{\text {large }}, \mathrm{kJ} / \mathrm{mol} & \ln k_{0}^{\text {small }}, \mathrm{s}^{-1} & \left\langle E_{\mathrm{a}}^{\text {small }}\right\rangle, \mathrm{kJ} / \mathrm{mol} & \sigma_{\text {small, }} \mathrm{kJ} / \mathrm{mol} \\ \text { F51 hydrated } & 25.4 \pm 1.5 & 23.9 \pm 3.1 & 1.9 \pm 0.4 & 19.6 \pm 0.9 & 1.4 \pm 0.3 \\ \text { F58 hydrated } & 23.9 \pm 1.7 & 20.8 \pm 3.5 & 2.0 \pm 0.4 & 18.0 \pm 0.7 & 3.25 \pm 0.6 \\ \text { F58 dry } & 29.0 \pm 2.5 & 36.5 \pm 4.0 & 3.0 \pm 0.5 & 21.4 \pm 0.7 & 6.9 \pm 1.2\end{array}$

fluctuations (Figure 4A). This pattern is reflected in the potential depicted in Figure 4B.

As discussed in the previous section, the nonexponentiality of the magnetization buildup curves is reflective of a distribution of conformers with different rate constants. The most logical description of this distribution within the concept of the energy landscape is to distinguish them by the different values of the activation energies. As elaborated in the Materials and Methods, from a technical standpoint the simulations are accomplished in the two-dimensional activation energy space denoted by the variables $E_{\mathrm{a}}^{\text {large }}$ and $E_{\mathrm{a}}^{\text {small }}$ (also defined in Figure $3 \mathrm{~B})$. The form of the distribution for the large-angle jumps is taken as Gaussian. However, for the small-angle jumps, we do not expect the average values of the activation energies to be much larger than the width of the distribution, and, therefore, we selected the gamma distribution, which excludes the negative values of the activation energies. The temperature dependence of $\beta$ is driven by the temperature dependence of the width of the distribution of the rate constants, which are directly related to the width of the distributions in the activation energies by $\sigma^{\text {large }} / T$ and $\sigma^{\text {small }} / T$. Thus, we see that for a fixed width of the distribution in the activation energy space, the distribution in the rate constants, in general, becomes wider as the temperature is lowered, which leads to a decrease in the values of $\beta$. This is consistent with the experimentally observed pattern. We note that the temperature dependence of $\beta$ can become more complicated around the temperature of the crossover between the two mechanisms, as discussed in Supporting Information SI3.

In the search for the value for the amplitude of the smallangle fluctuations, we fitted the data with small-angle jumps of $\alpha=5^{\circ}, 10^{\circ}, 20^{\circ}, 30^{\circ}$, and $40^{\circ}$. The data are consistent with the small-angle values of $5^{\circ}$ and $10^{\circ}$, but start to show discrepancies for larger values of $\alpha$ (Supporting Information SI4). The fitted parameters are weakly dependent on whether $5^{\circ}$ or $10^{\circ}$ are used, and we, thus, use $5^{\circ}$ as the lower limit of the small-angle jumps. The onset of the dominance of the small-angle fluctuation mechanism occurs when the temperature is lowered to $194 \mathrm{~K}$ for F58 and $200 \mathrm{~K}$ for F51. We defined the crossover temperature between the two mechanisms $\left(T_{\mathrm{c}}\right)$ as the point at which the relaxation times corresponding to the large- and small-angle jump mechanisms are equal for the mean values of $\left\langle E_{\mathrm{a}}^{\text {large }}\right\rangle$ and $\left\langle E_{\mathrm{a}}^{\text {small }}\right\rangle$ (Supporting Information SI3).

The comparison between the simulated and experimental relaxation anisotropies is one of the ways to validate the model. As indicated above, we did not observe any anisotropy within the precision of our measurements, which puts a constraint on the simulated anisotropy values to be below about $15 \%$. We defined the relaxation anisotropy as the deviation of the relaxation times from $T_{1}^{\text {eff }}$ at the $\pm 60 \mathrm{kHz}$ spikelets. The simulated value of the anisotropy is in the range of $2-8 \%$ for low temperatures at which the small-angle jumps dominate and in the range of $5-15 \%$ for high temperatures dominated by the ring-flipping motions.
The fitted values of the activation energies (Table 1) are in the range of $21-23 \mathrm{~kJ} / \mathrm{mol}$ for large-angle jumps and $3-5 \mathrm{~kJ} /$ mol for small-angle jumps. For both modes, the widths are around $1.5-2 \mathrm{~kJ} / \mathrm{mol}$. It is interesting that these widths are very similar to what was found for the flanking methyl groups of V50, L61, L69, and L75, supporting the hypothesis of the concerted fluctuations in the core. The fitted values of the activation energies are comparable for the F58 and F51 rings, with the values for F51 being slightly higher. There is no evidence of conformational exchange between the substates with different activation energy values for phenylalanine dynamics. Fast conformational exchange would average out the differential $T_{1}$ contribution of the multiple conformers and render the magnetization buildup monoexponential. At the opposite limit, at which the rate constant of the conformational exchange is much less than $T_{1}^{\text {eff }}$, the distribution appears to be static. We thus conclude that any possible conformational exchange processes between the substates at the phenylalanine sites should be slower than about $1.5 \mathrm{~s}$ at room temperature. This number is about 5 times larger than that estimated for some of the flanking methyl groups.

Note that similar motional modes have been observed for phenylene rings of glassy polymers such as bisphenol A polycarbonate and bisphenol A polysulfones as probed by neutron scattering studies. ${ }^{67,68}$ The dynamics was described by the two main modes of ring-flips and relatively small-angle overdamped oscillations, with individual distributions of activation energy barriers for each mode. This underscores a fundamental similarity between proteins and polymers. However, one would expect marked differences in the parameters, especially in the characteristic widths of the distributions, as the hydrophobic core is expected to have a higher degree of order. Indeed, the widths of the distributions in the polymers are on the order of $10 \mathrm{~kJ} / \mathrm{mol}$ for both the oscillations and the flips, which is several times larger than in the protein. In addition, the oscillations are of much higher amplitude from $30^{\circ}$ to $50^{\circ}$, as opposed to $5-10^{\circ}$ in the protein. Also, the experimental results ${ }^{61,62}$ suggested that $k_{0}^{\text {large }}=k_{0}^{\text {small }}$, which is in favor of the diffusion origin of the ring-flipping motion in polymers, contrary to our results on the protein, for which a strong collision scenario related to the concerted changes of the hydrophobic core environment is more likely.

In our model (Figure 3), we suggest that both small- and large-angle jumps occur around the same dihedral angle $\chi_{2}$, as this is the simplest assumption. Small-angle jumps are not subject to strong suppression by steric interactions as are largeangle jumps. Therefore, there is no a priori reason to limit small-angle motions to rotations around the phenyl axis. Smallangle jumps will contribute comparably to the relaxation process independent of their exact geometry with a caveat that for motions that involve a change in the direction of the $\mathrm{C}^{\beta}-\mathrm{C}^{\gamma}$ axis, the $\mathrm{D}^{\zeta}$ deuteron will contribute to the relaxation on the same time scale as the other phenyl ring deuterons.

A wide range of values between 27 and $155 \mathrm{~kJ} / \mathrm{mol}$ have been reported in the literature for the activation energies of the 

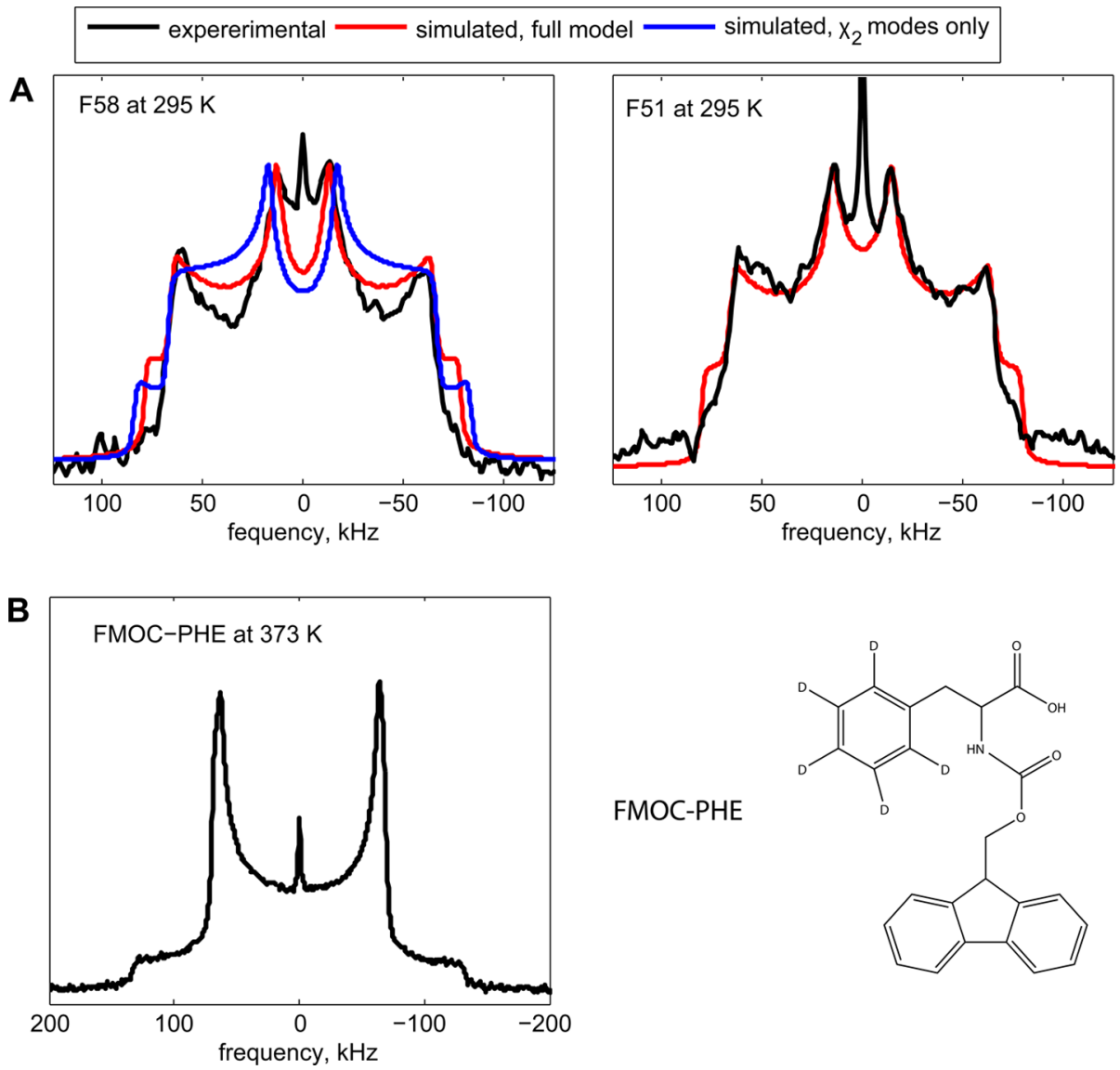

Figure 7. (A) Experimental deuteron NMR line shapes (black) acquired with the quadrupolar echo acquisition scheme at $295 \mathrm{~K}$ are overlaid with the simulated line shapes. The red lines correspond to the full motional model with fluctuations around both the $\chi_{1}$ and the $\chi_{2}$ angles and about $1^{\circ}$ deviations from the ideal ring geometry, as discussed in the text. The blue line, shown for the F58 residue, was simulated using only the modes of motions around the $\chi_{2}$ angle (both the large-angle flips and the small-angle fluctuations) with the parameters fixed from the relaxation data. An ideal ring geometry of $60^{\circ}$ was used in this case. The narrow peak at the center of the spectra is the signal from HOD. (B) Line shape spectrum for the FMOC-Phe-ring- $\mathrm{d}_{5}$ amino acid at $373 \mathrm{~K}$ and its chemical structure.

ring-flipping motions of tyrosine and phenylalanine in peptides and proteins. ${ }^{18-22,32-34}$ This wide range indicates that the ringflipping motion is highly dependent on the environment. For example, an insightful investigation by Bajaj et al. ${ }^{32}$ compared two structurally similar peptides that display very different ringflipping rates attributed to differences in packing. Our central values of $21-23 \mathrm{~kJ} / \mathrm{mol}$ are at the lowest end of this range and indicate a relatively flexible environment in the hydrophobic core of HP36.

IV. Line Shapes Report on Additional Fluctuations around the $\chi_{1}$ Angle. Experimental quadrupolar echo line shapes provide an additional independent test of the validity of the motional model and can point to aspects that need further refinement. The quadrupole echo acquisition scheme with one echo is better for this purpose as compared to the multipleecho detection, as it provides more detailed information. Because of the low signal-to-noise ratio and increasingly large relaxation delays at low temperatures, reliable experimental line shapes were collected only at $295 \mathrm{~K}$ (Figure 7). The experimental line shapes display two types of singularities: the inner ones originating from the perpendicular edges (horns) of the flip-averaged deuteron tensors and the outer ones due to the perpendicular edges of the static tensors.

When we compare the experimental line shapes with the model developed on the basis of the relaxation data (shown in Figure 7A by the blue line for F58), we obtain good agreement in the overall pattern. The agreement in the relative heights of the inner and outer singularities indicates that the overall distribution of ring-flip rates is captured well in this model. Note that the peak at zero frequency corresponds to the residual HOD signal. However, two features in the experimental line shapes require further refinements of the model: (i) the positions of the inner horn relative to the outer horns are narrowed in the experimental spectrum as compared to the simulated one and (ii) the depth of the valleys between the inner and outer singularities is more pronounced in the experimental spectrum as compared to the simulated one.

To address the first feature, we note that the agreement with the experimental line shape cannot be achieved by the introduction of any additional motion of the ring axis, as this would lead to an overall narrowing of the powder pattern. Two possible explanations can selectively narrow the position of the inner horns. The first one involves the adjustment of the intrinsic asymmetry parameter. An alternative explanation is a slight deviation from the ideal ring geometry. Several sets of tensor parameters have been reported for crystalline phenylalanine amino acid. For example, Gall et al. ${ }^{26}$ and Kinsey et al. ${ }^{69}$ reported a $C_{\mathrm{q}}$ of $180 \mathrm{kHz}$ and $\eta=0.05$ based on line shape data at 100 and $298 \mathrm{~K}$, respectively. Alternatively, Hiyama et al. ${ }^{27}$ reported a $C_{\mathrm{q}}=171 \mathrm{kHz}$ and $\eta<0.02$ in conjunction with deviations from the ideal ring geometry based on the spectral data in the range of 293-421 K. Our experimental quadrupole 

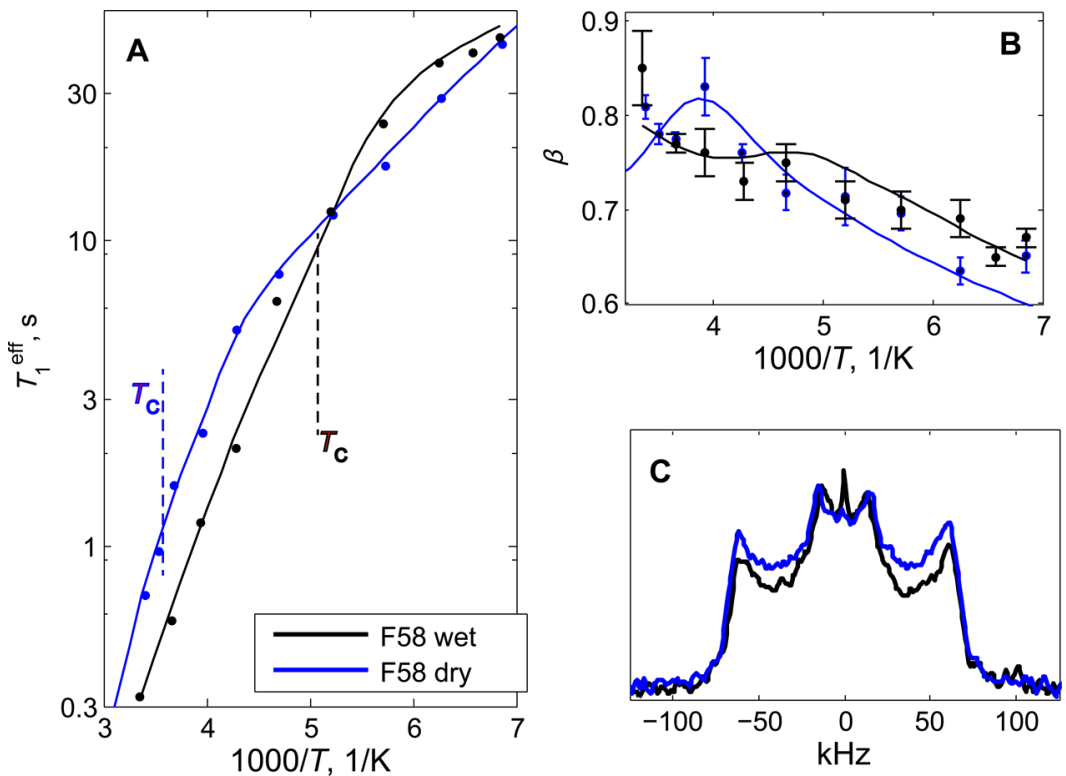

Figure 8. Relaxation and line shape data for the F58-ring- $\mathrm{d}_{5}$ site in the hydrated (black lines) and dry (blue lines) powder states. (A) Plots of $T_{1}^{\text {eff }}$ versus $1000 / T$ on the semilog scale. The errors are within the size of the symbol. The dotted lines indicate the crossover temperatures $T_{c}$. (B) $\beta$ versus 1000/T. The data are shown for the temperature range between 295 and $145 \mathrm{~K}$ and the magnetic field strength of $17.6 \mathrm{~T}$. (C) Quadrupolar echo line shapes at $295 \mathrm{~K}$. The spectra are scaled to yield identical heights of the inner singularities (horns).

echo data for both residues at $295 \mathrm{~K}$ can be fitted equally well with either $C_{\mathrm{q}}=180 \mathrm{kHz}, \eta=0$, and an about $1^{\circ}$ difference from the ideal ring geometry or with about $170 \mathrm{kHz}, \eta=0.06-$ 0.07 , and the ideal ring geometry. To distinguish between these two possibilities, we turn to our low temperature spectra, which were collected with the QCPMG acquisition scheme. While the spectra are not sensitive to the range of $\eta$ between 0 and 0.07 , they point toward a value of the quadrupolar coupling constant of $180 \mathrm{kHz}$ as opposed to $170 \mathrm{kHz}$ (Supporting Information SI5). We, thus, keep the following final parameters: $C_{\mathrm{q}}=180$ $\mathrm{kHz}, \eta=0$, and the angles between either the $\mathrm{C}^{\delta}-\mathrm{D}$ or the $\mathrm{C}^{\varepsilon}-$ $\mathrm{D}$ bonds and the $\mathrm{C}^{\beta}-\mathrm{C}^{\gamma}$ axis of $58.5^{\circ}$ for F58 and $59^{\circ}$ for F51. As can be seen from Figure $7 \mathrm{~A}$, the relative positions of the inner and outer horns agree well in the experimental and simulated spectra, as shown by the red line.

The second discrepancy between the simulations and experiment, the depth of the valleys between the inner and outer singularities, suggests additional asymmetric motions on the time scale of the quadrupolar coupling constant. When we include an additional motional mode corresponding to restricted diffusion around the axis perpendicular to the direction of the flips, the quality of the fit is significantly improved (Figure 7A, red line). This motional mode corresponds to restricted rotations around the $\chi_{1}$ angle and represents the fluctuations of this angle within the rotameric well. It has been modeled as the diffusion of the $\mathrm{C}^{\beta}-\mathrm{C}^{\gamma}$ axis along a restricted arc (Figure 4A). The best-fit parameter for the length of the arc, representing the variations in $\chi_{1}$, is $30^{\circ}$, and the diffusion rate is $760 \mathrm{rad}^{2} / \mathrm{s}$ for both residues. The amplitude of $30^{\circ}$ requires either relatively low packing density or the overall flexibility of the core to accommodate these motions. Thus, this result supports the conclusion obtained on the basis of the relaxation data, which pointed toward a relatively flexible core based on the values of the activation energies for the ring-flipping motion. However, the core environment is not so unconstrained as to allow for largeamplitude jumps of the ring axes, such as rotameric transitions that do not reproduce the experimental line shape data. This is consistent with the recent studies by $\mathrm{Li}$ et al., ${ }^{36}$ which showed that rotameric transition does not exist in a free (i.e., nonsurface bound) peptide.

We also looked at the dependence of the line shapes on the interpulse delay $\tau$ in the quadrupolar echo experiment $\left(90_{x}^{0}-\tau-\right.$ $\left.90_{y}^{0}\right)$. As noted by Schadt et al., ${ }^{70}$ the dependence of the line shapes on this delay enables us to validate the width of the distribution of the conformers with different ring-flip rates. If the distribution in the rate constants is wider than two decades, then the corresponding line shapes are no longer dependent on the value of $\tau$. In contrast, our relatively narrow width of the distribution preserves the dependence on $\tau$. Experimental and simulated line shapes as a function of $\tau$ delays are presented in Supporting Information SI6, and they demonstrate good agreement. Further validation of the model for the description of the line-shapes could be obtained by magic-angle spinning experiments at lower temperatures.

It is also interesting to compare the dynamics of the phenyl rings in the protein core with the behavior of the ring in the FMOC-phenylalanine amino acid. The bulky hydrophobic FMOC group creates hydrophobic contacts for packing, yet lacks the complexity of the core. The line shapes of FMOC-Phe are completely rigid, even at $373 \mathrm{~K}$ (Figure $7 \mathrm{~B}$ ). This result underscores that the environment of the protein core can allow for much more enhanced dynamics as compared to general hydrophobic-type packing.

V. Hydration Dependence of the Dynamics and Manifestation of the Protein Dynamical Transition. To assess how the motions depend on hydration, we compared both the relaxation and the line shape data for the side chain of F58 in the hydrated and dry powder states. For the dry sample, the data were obtained only at one field of $17.6 \mathrm{~T}$ in the $295-$ $145 \mathrm{~K}$ range (Figure $8 \mathrm{~A}$ ). The value of $T_{1}^{\mathrm{eff}}$ is about 2.5 higher at room temperature for the dry protein, and the fitted value of the activation energy for the ring-flip motions is also significantly larger in the dry sample, with an increase of 
about $15 \mathrm{~kJ} / \mathrm{mol}$ (Table 1 ). The value of $15 \mathrm{~kJ} / \mathrm{mol}$ can be viewed as the magnitude of change in the local potential of the hydrophobic core environment induced by dehydration. Smallamplitude fluctuations in the dry protein take place under this modified environment, and the activation energy for these fluctuations is somewhat different (by $3.6 \mathrm{~kJ} / \mathrm{mol}$ ) but well within the $15 \mathrm{~kJ} / \mathrm{mol}$ magnitude. It is also important that the widths of the distributions in the activation energies are of the same order of magnitude for both the $\pi$-flips and the smallangle fluctuations $(2-3 \mathrm{~kJ} / \mathrm{mol})$, reinforcing the notion that the energy landscape of the small-angle fluctuations adjusts to changes in the overall potential caused by dehydration.

The effect of dehydration on the rate constants themselves is shown in Figure 9 for the entire temperature range, using the

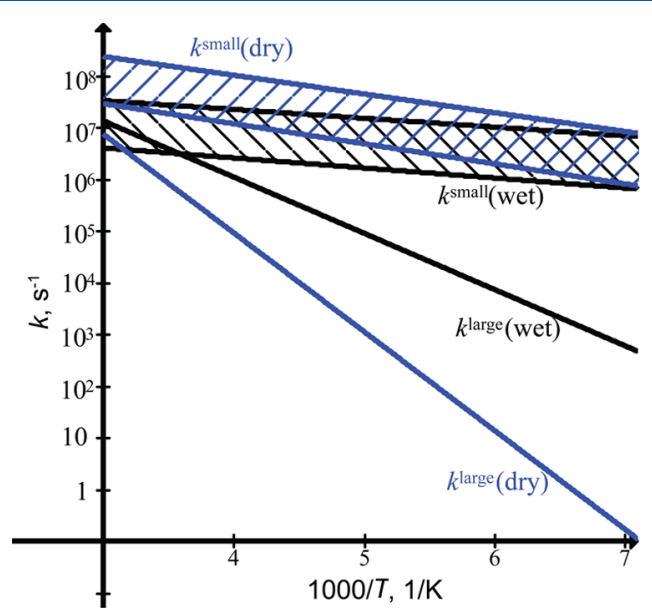

Figure 9. Rate constants $k^{\text {large }}$ and $k^{\text {small }}$ over the temperature range of 295-145 K for the F58-ring- $\mathrm{d}_{5}$ site in the hydrated (black) and dry (blue) states. The hatch bands represent the limits of uncertainties in $k^{\text {small }}$ introduced by uncertainty in $\alpha$ in the $4-11^{\circ}$ range.

parameters listed in Table 1 . Note that for the small-angle fluctuations, the rate constant $k^{\text {small }}$ obtained from the fits is strongly influenced by the uncertainty in the value of angle $\alpha$. The bands in Figure 9 represent the uncertainties in $k^{\text {small }}$ when $\alpha$ is taken in the range of $4-11^{\circ}$. While a pronounced effect of dehydration is observed for the ring-flipping motion, the rate constants for small-angle fluctuations are similar for dry and wet proteins within the uncertainties. Looking at the relative intensities of singularities in the line shape data at $295 \mathrm{~K}$ (Figure 8C), it is also evident that the distribution of the conformers is shifted toward slower ring-flip rates in the dry sample. The line shape data are not sensitive to small-angle fluctuations.

Another interesting feature in the comparison between the relaxation behavior of the dry and wet proteins is that the crossover from the dominance of the small-angle fluctuations to the ring-flipping motions occurs at a much higher temperature for the dry sample. The estimated temperature of the crossover is around $278 \mathrm{~K}$ for the dry protein as compared to about 194 $\mathrm{K}$ for the wet protein (Supporting Information SI3). We also note that due to the larger differences in the values of $\left\langle E_{\mathrm{a}}^{\mathrm{large}}\right\rangle$ and $\left\langle E_{\mathrm{a}}^{\text {small }}\right\rangle$ in the dry protein, the nonmonotonous temperature dependence of $\beta$ is expected to be more pronounced around the crossover temperature.

Dynamical transitions in proteins are characterized by the onset of large-scale motions upon heating from cryogenic temperatures. ${ }^{71}$ They remain a topic of very active inves- tigations by means of neutron scattering, NMR spectroscopy, and other techniques. ${ }^{5,71-77}$ Notably, the crossover point between the small-amplitude fluctuations and ring-flipping motions in the hydrated HP36 protein is close to the temperature of the dynamical transition for the backbone dynamic mode recently studied by Kämpf et al. ${ }^{72}$ by deuterium NMR relaxation and molecular dynamics simulations. Their work also pointed to the importance of the role of the hydration shell in inducing these motions. In addition, Lewandowski et al. ${ }^{75}$ recently emphasized the importance of the hydration shell for inducing backbone and side chain motion with activation energies above $20 \mathrm{~kJ} / \mathrm{mol}$. Interestingly, the temperature of onset for these motions reported for the hydrated microcrystalline GB1 protein is $195 \mathrm{~K}$, which coincides with the crossover temperature for HP36 in the hydrated state.

In contrast to the present results on phenylalanine side chains, our previous investigation of the effect of hydration on the relaxation behavior of a core methyl-bearing side chain of L69 showed identical results for dry and hydrated proteins. ${ }^{42}$ Thus, our data suggest that the ring-flipping motions in aromatic residues is hydration-dependent, whereas the threesite jumps of methyl groups do not depend on hydration.

For the ring-flipping motion to occur, the hydrophobic core has to be able to adjust to the instantaneous rotational state of the ring in order to release steric hindrance, which otherwise precludes the motions of the bulky ring. ${ }^{78,79}$ Hydration likely aids in rendering the core softer, resulting in more efficient adjustments of the environment. This implies propagation of the hydration effect from the protein surface into the hydrophobic core. ${ }^{80,81}$ Fast three-site methyl jumps do not require large adjustments in the core due to the much smaller size of the methyl groups as compared to the aromatic groups, which can be the reason for their hydration-independent behavior. However, the motions of the methyl axes, which involve large-angle rotameric rearrangements, are dependent on hydration. ${ }^{44}$ In analogy with the ring-flipping motion, largeangle rearrangements of the entire methyl-bearing side-chain of leucine would involve releasing the steric hindrance of neighboring atoms. On the other hand, the existence of the distributions of activation energies with similar widths for both the methyl jumps and the phenyl flips implies additional slow concerted core fluctuations (slower than the ring-flipping motion) that give rise to these distributions. Molecular dynamics simulations in the solid state are necessary to obtain further molecular insights into all of these different motional modes inside the core and the extent of correlations among them. Results on phenylalanine side-chains presented in this work demonstrate that aromatic residues can be important markers of protein dynamical transitions.

\section{CONCLUSION}

This work presents one of the most detailed quantitative characterizations of aromatic side chain motions in the solid state. The side chains of F58 and F51 participate in two main motional modes, with ring-flips dominating at high temperatures and small-angle jumps dominating at low temperatures. The ring-flipping motion occurs in the strong collision limit as opposed to restricted diffusion. The onset of the dominance of the small-angle jump mechanism occurs at about $200-195 \mathrm{~K}$ in the hydrated protein and shifts to a much higher temperature of about $280 \mathrm{~K}$ in the dry protein. The crossover point between the small-amplitude fluctuations and ring-flipping motions in 
the hydrated HP36 protein is close to the temperature of the dynamical transition temperature reported for the high activation energy motional modes of side chains and backbones in other proteins. The $80 \mathrm{~K}$ change in the onset of ring-flipping motions between the hydrated and dry proteins points to the role of solvent in softening the core and emphasizes the role of aromatic residues as useful markers of protein dynamical transitions.

The observed nonexponentiality of the magnetization buildup curves has led to the model involving the distributions of conformers distinguished by their values of activation energies for the ring-flips and small-angle fluctuations. The central values of the distributions are similar for the two residues and fall in the ranges of $21-23$ and $3-5 \mathrm{~kJ} / \mathrm{mol}$ for the ring-flips and small-angle jumps, respectively. These activation energies for the ring-flipping motion are among the smallest reported for phenylalanine and tyrosine side chains in peptides and proteins and indicate a relatively flexible core. Dehydration of the protein leads to the shift of the distribution toward a lot more rigid conformers for the ring-flipping motion, manifesting in a $15 \mathrm{~kJ} / \mathrm{mol}$ increase in the central value of the activation energy. The line shape data at $295 \mathrm{~K}$ suggest the presence of one dominant rotameric configuration of the side chains without any rotameric interconversions on $\mu \mathrm{s}$-ms time scales. The widths of the rotameric well for the $\chi_{1}$ angle are about $30^{\circ}$ for both residues, also supporting the notion of a relatively flexible core environment in the hydrated protein.

This study complements our previous investigations of the dynamics of hydrophobic core methyl groups. The combined analysis of aromatic and methyl sites allows for a very thorough view of the free energy landscape in the hydrophobic core. Within our model, the heterogeneity of the local free energy landscape is characterized by the widths of the distribution of the activation energies. These widths are on the order of 1.5-2 $\mathrm{kJ} / \mathrm{mol}$ for both modes of motions in the phenylalanine residues as well as for the methyl rotational jumps. The presence of the distributions at multiple sites strongly suggests the existence of slow motional modes common to the entire core. The presented experimental results set the stage for molecular dynamic simulations in the solid state and at low temperatures, which can elucidate the structural details of the conformational substates that give rise to the distributions.

\section{ASSOCIATED CONTENT}

\section{S Supporting Information}

The Supporting Information is available free of charge on the ACS Publications website at DOI: 10.1021/acs.jpcb.5b09299.

SI1, ${ }^{15} \mathrm{~N}$ backbone chemical shifts for several core phenylalanine and leucine residues; SI2, full analytical description of the model used for the description of the longitudinal relaxation; SI3, analysis of the crossover point for the two relaxation mechanisms; SI4, simulations of $T_{1}^{\text {eff }}$ and $\beta$ for different values of the small-angle jumps parameter $\alpha$; SI5, quadrupolar coupling constant analysis based on the QCPMG line shapes at low temperatures; and SI6, the dependence of the experimental and simulated quadrupole echo line shapes on the interpulse delay (PDF)

\section{AUTHOR INFORMATION}

\section{Corresponding Author}

*E-mail: liliya.vugmeyster@ucdenver.edu.

\section{Notes}

The authors declare no competing financial interest.

\section{ACKNOWLEDGMENTS}

Support for this research was received from the National Institutes of Health Grant 1R15 GM111681-02 to L.V., National Science Foundation Grants MCB-1122154 to L.V. and D.O., CHE-0713819 and CHE-1012344 to R.L.V. and G.L.H., and National Institutes of Health Grant R15GM097605-01 to R.L.V. Part of this research was performed using EMSL, a national scientific user facility sponsored by the Department of Energy's Office of Biological and Environmental Research located at Pacific Northwest National Laboratory.

\section{REFERENCES}

(1) Creighton, T. E. Proteins: Structures and Molecular Properties; W. H. Freeman and Company: New York, 1993.

(2) Onuchic, J. N.; LutheySchulten, Z.; Wolynes, P. G. Theory of Protein Folding: The Energy Landscape Perspective. Annu. Rev. Phys. Chem. 1997, 48, 545-600.

(3) Igumenova, T. I.; Frederick, K. K.; Wand, A. J. Characterization of the Fast Dynamics of Protein Amino Acid Side Chains Using NMR Relaxation in Solution. Chem. Rev. 2006, 106, 1672-1699.

(4) Krushelnitsky, A.; Reichert, D. Solid-State NMR and Protein Dynamics. Prog. Nucl. Magn. Reson. Spectrosc. 2005, 47, 1-25.

(5) Lee, A. L.; Wand, A. J. Microscopic Origins of Entropy, Heat Capacity and the Glass Transition in Proteins. Nature 2001, 411, 501504.

(6) Mittermaier, A. K.; Kay, L. E. Observing Biological Dynamics at Atomic Resolution Using NMR. Trends Biochem. Sci. 2009, 34, 601611.

(7) Vold, R. L.; Vold, R. R. Deuterium Relaxation in Molecular Solids. In Advances in Magnetic and Optical Resonance; Warren, W., Ed.; Acadenic Press: San Diego, CA, 1991; pp 85-171.

(8) Xue, Y.; Pavlova, M. S.; Ryabov, Y. E.; Reif, B.; Skrynnikov, N. R. Methyl Rotation Barriers in Proteins from H-2 Relaxation Data. Implications for Protein Structure. J. Am. Chem. Soc. 2007, 129, 68276838.

(9) Meints, G. A.; Miller, P. A.; Pederson, K.; Shajani, Z.; Drobny, G. Solid-State Nuclear Magnetic Resonance Spectroscopy Studies of Furanose Ring Dynamics in the DNA Hhal Binding Site. J. Am. Chem. Soc. 2008, 130, 7305-7314.

(10) Olsen, G. L.; Bardaro, M. F.; Echodu, D. C.; Drobny, G. P.; Varani, G. Hydration Dependent Dynamics in Rna. J. Biomol. NMR 2009, 45, 133-142.

(11) McDermott, A.; Polenova, T. Solid State NMR: New Tools for Insight into Enzyme Function. Curr. Opin. Struct. Biol. 2007, 17, 617622.

(12) Fu, Y. N.; Kasinath, V.; Moorman, V. R.; Nucci, N. V.; Hilser, V. J.; Wand, A. J. Coupled Motion in Proteins Revealed by Pressure Perturbation. J. Am. Chem. Soc. 2012, 134, 8543-8550.

(13) Weininger, U.; Respondek, M.; Low, C.; Akke, M. Slow Aromatic Ring Flips Detected Despite near-Degenerate NMR Frequencies of the Exchanging Nuclei. J. Phys. Chem. B 2013, 117, 9241-9247.

(14) Kasinath, V.; Valentine, K. G.; Wand, A. J. A C-13 Labeling Strategy Reveals a Range of Aromatic Side Chain Motion in Calmodulin. J. Am. Chem. Soc. 2013, 135, 9560-9563.

(15) Frey, M. H.; Diverdi, J. A.; Opella, S. J. Dynamics of Phenylalanine in the Solid-State by NMR. J. Am. Chem. Soc. 1985, 107, 7311-7315.

(16) Gall, C. M.; Cross, T. A.; Diverdi, J. A.; Opella, S. J. Protein Dynamics by Solid-State NMR - Aromatic Rings of the Coat Protein in Fd Bacteriophage. Proc. Natl. Acad. Sci. U. S. A. 1982, 79, 101-105.

(17) Zhang, H. M.; Bryant, R. G. Characterization of Enzyme-Bound Ligand Dynamics by Solid-State NMR in the Presence of Ligand- 
Exchange - L-Phenylalanine on Carboxypeptidase-A. Biophys. J. 1995, 68, 303-311.

(18) Hattori, M.; Li, H.; Yamada, H.; Akasaka, K.; Hengstenberg, W.; Gronwald, W.; Kalbitzer, H. R. Infrequent Cavity-Forming Fluctuations in Hpr from Staphylococcus Carnosus Revealed by Pressure- and Temperature-Dependent Tyrosine Ring Flips. Protein Sci. 2004, 13, 3104-3114.

(19) Otting, G.; Liepinsh, E.; Wuthrich, K. Disulfide Bond Isomerization in Bpti and Bpti(G36s) - an NMR-Study of Correlated Mobility in Proteins. Biochemistry 1993, 32, 3571-3582.

(20) Rao, D. K.; Bhuyan, A. K. Complexity of Aromatic Ring-Flip Motions in Proteins: Y97 Ring Dynamics in Cytochrome C Observed by Cross-Relaxation Suppressed Exchange NMR Spectroscopy. J. Biomol. NMR 2007, 39, 187-196.

(21) Campbell, I. D.; Dobson, C. M.; Moore, G. R.; Perkins, S. J.; Williams, R. J. P. Temperature-Dependent Molecular-Motion of a Tyrosine Residue of Ferrocytochrome-C. FEBS Lett. 1976, 70, 96100.

(22) Wagner, G.; Demarco, A.; Wuthrich, K. Dynamics of Aromatic Amino-Acid Residues in Globular Conformation of Basic Pancreatic Trypsin-Inhibitor (Bpti) 0.1. H-1 NMR-Studies. Biophys. Struct. Mech. 1976, 2, 139-158.

(23) Kasinath, V.; Fu, Y. N.; Sharp, K. A.; Wand, A. J. A Sharp Thermal Transition of Fast Aromatic-Ring Dynamics in Ubiquitin. Angew. Chem., Int. Ed. 2015, 54, 102-104.

(24) Wagner, G. Activation Volumes for the Rotational Motion of Interior Aromatic Rings in Globular-Proteins Determined by HighResolution H-1-NMR at Variable Pressure. FEBS Lett. 1980, 112, 280-284.

(25) Vold, R. R. Deuterium NMR Studies of Dynamics in Solids and Liquid Crystals. In Nuclear Magnetic Resonance Probes of Molecular Dynamics; Tycko, R., Ed.; Kluwer Academic Publishers: Dordrecht, 1994; pp 27-112.

(26) Gall, C. M.; Diverdi, J. A.; Opella, S. J. Phenylalanine Ring Dynamics by Solid-State H-2 NMR. J. Am. Chem. Soc. 1981, 103, 5039-5043.

(27) Hiyama, Y.; Silverton, J. V.; Torchia, D. A.; Gerig, J. T.; Hammond, S. J. Molecular-Structure and Dynamics of Crystalline Para-Fluoro-D,L-Phenylalanine - a Combined X-Ray NMR Investigation. J. Am. Chem. Soc. 1986, 108, 2715-2723.

(28) Schulz, M.; Vanderest, A.; Rossler, E.; Kossmehl, G.; Vieth, H. M. H-2 NMR-Study of Benzene-D6 in a Liquid-Crystalline Polysiloxane. Macromolecules 1991, 24, 5040-5045.

(29) Hiraoki, T.; Kogame, A.; Nishi, N.; Tsutsumi, A. Deuterium NMR Studies on Phenyl Ring Dynamics of Poly(L-Phenyl Alanine). J. Mol. Struct. 1998, 441, 243-250.

(30) Henrichs, P. M.; Nicely, V. A.; Fagerburg, D. R. Sub-Tg Dynamic Processes in Amorphous Solids - a Deuterium and C-13 NMR-Study of Poly(Phenylene Sulfide). Macromolecules 1991, 24, 4033-4037.

(31) Naito, A.; Iizuka, T.; Tuzi, S.; Price, W. S.; Hayamizu, K.; Saito, $\mathrm{H}$. Phenyl Ring Dynamics of the Insulin Fragment Gly-Phe-Phe(B23B25) by Solid-State Deuterium NMR. J. Mol. Struct. 1995, 355, 55-60.

(32) Bajaj, V. S.; van der Wel, P. C. A.; Griffin, R. G. Observation of a Low-Temperature, Dynamically Driven Structural Transition in a Polypeptide by Solid-State NMR Spectroscopy. J. Am. Chem. Soc. 2009, 131, 118-128.

(33) Rice, D. M.; Meinwald, Y. C.; Scheraga, H. A.; Griffin, R. G. Tyrosyl Motion in Peptides - H-2 NMR Line-Shapes and Spin-Lattice Relaxation. J. Am. Chem. Soc. 1987, 109, 1636-1640.

(34) Kamihira, M.; Naito, A.; Tuzi, S.; Saito, H. Phenyl Ring Dynamics of Enkephalin Molecules and Behavior of Bound Solvents in the Crystalline States by H-2 NMR Spectroscopy. J. Phys. Chem. A 1999, 103, 3356-3363.

(35) Rice, D. M.; Wittebort, R. J.; Griffin, R. G.; Meirovitch, E.; Stimson, E. R.; Meinwald, Y. C.; Freed, J. H.; Scheraga, H. A. Rotational Jumps of the Tyrosine Side-Chain in Crystalline Enkephalin - H-2 NMR Line-Shapes for Aromatic Ring Motion in Solids. J. Am. Chem. Soc. 1981, 103, 7707-7710.
(36) Li, K.; Emani, P. S.; Ash, J.; Groves, M.; Drobny, G. P. A Study of Phenylalanine Side-Chain Dynamics in Surface-Adsorbed Peptides Using Solid-State Deuterium NMR and Rotamer Library Statistics. J. Am. Chem. Soc. 2014, 136, 11402-11.

(37) Larsen, F. H.; Jakobsen, H. J.; Ellis, P. D.; Nielsen, N. C. HighField Qcpmg-Mas NMR of Half-Integer Quadrupolar Nuclei with Large Quadrupole Couplings. Mol. Phys. 1998, 95, 1185-1195.

(38) McKnight, C. J.; Doering, D. S.; Matsudaira, P. T.; Kim, P. S. A Thermostable 35-Residue Subdomain within Villin Headpiece. J. Mol. Biol. 1996, 260, 126-134.

(39) McKnight, C. J.; Matsudaira, P. T.; Kim, P. S. NMR Structure of the 35-Residue Villin Headpiece Subdomain. Nat. Struct. Biol. 1997, 4, $180-184$.

(40) Chiu, T. K.; Kubelka, J.; Herbst-Irmer, R.; Eaton, W. A.; Hofrichter, J.; Davies, D. R. High-Resolution X-Ray Crystal Structures of the Villin Headpiece Subdomain, an Ultrafast Folding Protein. Proc. Natl. Acad. Sci. U. S. A. 2005, 102, 7517-7522.

(41) Vugmeyster, L.; Tien, D.; Ostrovsky, D.; Fu, R. Effect of Subdomain Interactions on Methyl Group Dynamics in the Hydrophobic Core of Villin Headpiece Protein. Protein Sci. 2014, 23, 145156.

(42) Vugmeyster, L.; Ostrovsky, D.; Penland, K.; Hoatson, G. L.; Vold, R. L. Glassy Dynamics of Protein Methyl Groups Revealed by Deuteron NMR. J. Phys. Chem. B 2013, 117, 1051-1061.

(43) Vugmeyster, L.; Ostrovsky, D.; Lipton, A. S. Origin of Abrupt Rise in Deuteron NMR Longitudinal Relaxation Times of Protein Methyl Groups Below 90 K. J. Phys. Chem. B 2013, 117, 6129-6137.

(44) Vugmeyster, L.; Ostrovsky, D.; Khadjinova, A.; Ellden, J.; Hoatson, G. L.; Vold, R. L. Slow Motions in the Hydrophobic Core of Chicken Villin Headpiece Subdomain and Their Contributions to Configurational Entropy and Heat Capacity from Solid-State Deuteron NMR Measurements. Biochemistry 2011, 50, 10637-10646.

(45) Vugmeyster, L.; Ostrovsky, D.; Ford, J. J.; Lipton, A. S. Freezing of Dynamics of a Methyl Group in a Protein Hydrophobic Core at Cryogenic Temperatures by Deuteron NMR Spectroscopy. J. Am. Chem. Soc. 2010, 132, 4038-4039.

(46) Vugmeyster, L.; Ostrovsky, D.; Ford, J. J.; D, B. S.; Lipton, A. S.; Hoatson, G. L.; Vold, R. L. Probing the Dynamics of a Protein Hydrophobic Core by Deuteron Solid-State Nuclear Magnetic Resonance Spectroscopy. J. Am. Chem. Soc. 2009, 131, 13651-13658.

(47) Wood, K.; Gallat, F. X.; Otten, R.; van Heel, A. J.; Lethier, M.; van Eijck, L.; Moulin, M.; Haertlein, M.; Weik, M.; Mulder, F. A. A. Protein Surface and Core Dynamics Show Concerted HydrationDependent Activation. Angew. Chem., Int. Ed. 2013, 52, 665-668.

(48) Khodadadi, S.; Pawlus, S.; Sokolov, A. P. Influence of Hydration on Protein Dynamics: Combining Dielectric and Neutron Scattering Spectroscopy Data. J. Phys. Chem. B 2008, 112, 14273-14280.

(49) Krushelnitsky, A.; Zinkevich, T.; Mukhametshina, N.; Tarasova, N.; Gogolev, Y.; Gnezdilov, O.; Fedotov, V.; Belton, P.; Reichert, D. C-13 and N-15 NMR Study of the Hydration Response of T4 Lysozyme and Alpha B-Crystallin Internal Dynamics. J. Phys. Chem. B 2009, 113, 10022-10034.

(50) Rupley, J. A.; Gratton, E.; Careri, G. Water and GlobularProteins. Trends Biochem. Sci. 1983, 8, 18-22.

(51) Vugmeyster, L.; Osrovsky, D.; Fu, R. 15n Csa Tensors and 15n$1 \mathrm{~h}$ Dipolar Couplings of Protein Hydrophobic Core Residues Investigated by Static Solid-State NMR. J. Magn. Reson. 2015, 259, $225-231$.

(52) Lipton, A. S.; Heck, R. W.; Sears, J. A.; Ellis, P. D. Low Temperature Solid-State NMR Experiments of Half-Integer Quadrupolar Nuclides: Caveats and Data Analysis. J. Magn. Reson. 2004, $168,66-74$

(53) Beckmann, P. A.; Dybowski, C. A Thermometer for Nonspinning Solid-State NMR Spectroscopy. J. Magn. Reson. 2000, 146, 379-380.

(54) Duer, M. J. Solid-State NMR Spectroscopy; Blackwell Publishing Ltd.: Oxford, 2004.

(55) Torchia, D. A.; Szabo, A. Spin-Lattice Relaxation in Solids. J. Magn. Reson. 1982, 49, 107-121. 
(56) Meirovitch, E.; Nayeem, A.; Freed, J. H. Analysis of Protein Lipid Interactions Based on Model Simulations of Electron-Spin Resonance-Spectra. J. Phys. Chem. 1984, 88, 3454-3465.

(57) Meirovitch, E.; Liang, Z. C.; Freed, J. H. Protein Dynamics in the Solid State from H-2 NMR Line Shape Analysis: A Consistent Perspective. J. Phys. Chem. B 2015, 119, 2857-2868.

(58) Vold, R. L.; Hoatson, G. L. Effects of Jump Dynamics on Solid State Nuclear Magnetic Resonance Line Shapes and Spin Relaxation Times. J. Magn. Reson. 2009, 198, 57-72.

(59) Shapovalov, M. V.; Dunbrack, R. L., Jr. A Smoothed BackboneDependent Rotamer Library for Proteins Derived from Adaptive Kernel Density Estimates and Regressions. Structure 2011, 19, 844858.

(60) Hirschinger, J.; Miura, H.; Gardner, K. H.; English, A. D. Segmental Dynamics in the Crystalline Phase of Nylon-66 - Solid-State H-2 NMR. Macromolecules 1990, 23, 2153-2169.

(61) Sillescu, H. Heterogeneity at the Glass Transition: A Review. J. Non-Cryst. Solids 1999, 243, 81-108.

(62) Schnauss, W.; Fujara, F.; Hartmann, K.; Sillescu, H. Nonexponential H-2 Spin-Lattice Relaxation as a Signature of the Glassy State. Chem. Phys. Lett. 1990, 166, 381-384.

(63) Beckmann, P. A.; Schneider, E. Methyl Group Rotation, H-1 Spin-Lattice Relaxation in an Organic Solid, and the Analysis of Nonexponential Relaxation. J. Chem. Phys. 2012, 136, 054508.

(64) Lindsey, C. P.; Patterson, G. D. Detailed Comparison of the Williams-Watts and Cole-Davidson Functions. J. Chem. Phys. 1980, 73, 3348-3357.

(65) Kramers, H. A. Physica 1940, 7, 284-304.

(66) Vugmeyster, L.; Ostrovsky, D. Restricted Diffusion of Methyl Groups in Proteins Revealed by Deuteron NMR: Manifestation of Intra-Well Dynamics. J. Chem. Phys. 2014, 140, 075101.

(67) Arrese-Igor, S.; Arbe, A.; Alegria, A.; Colmenero, J.; Frick, B. Sub-T-G Dynamics in Polycarbonate by Neutron Scattering and Its Relation with Secondary Gamma Relaxation. J. Chem. Phys. 2005, 123, 014907.

(68) Arrese-Igor, S.; Arbe, A.; Alegria, A.; Colmenero, J.; Frick, B. Phenylene Ring Dynamics in Bisphenol-a-Polysulfone by Neutron Scattering. J. Chem. Phys. 2004, 120, 423-436.

(69) Kinsey, R. A.; Kintanar, A.; Oldfield, E. Dynamics of AminoAcid Side-Chains in Membrane-Proteins by High-Field Solid-State Deuterium Nuclear Magnetic-Resonance Spectroscopy - Phenylalanine, Tyrosine, and Tryptophan. J. Biol. Chem. 1981, 256, 90289036.

(70) Schadt, R. J.; Cain, E. J.; English, A. D. Simulation of OneDimensional H-2 NMR Line-Shapes. J. Phys. Chem. 1993, 97, 83878392.

(71) Doster, W. The Dynamical Transition of Proteins, Concepts and Misconceptions. Eur. Biophys. J. 2008, 37, 591-602.

(72) Kaempf, K.; Kremmling, B.; Vogel, M. Vanishing Amplitude of Backbone Dynamics Causes a True Protein Dynamical Transition: H2 NMR Studies on Perdeuterated C-Phycocyanin. Phys. Rev. E 2014, $89,032710$.

(73) Khodadadi, S.; Pawlus, S.; Roh, J. H.; Sakai, V. G.; Mamontov, E.; Sokolov, A. P. The Origin of the Dynamic Transition in Proteins. J. Chem. Phys. 2008, 128, 5-10.

(74) Doster, W. Dynamical Structural Distributions in Proteins. Phys. B 2006, 385-86, 831-834.

(75) Lewandowski, J. R.; Halse, M. E.; Blackledge, M.; Emsley, L. Direct Observation of Hierarchical Protein Dynamics. Science 2015, 348, 578-581.

(76) Magazu, S.; Migliardo, F.; Benedetto, A. Puzzle of Protein Dynamical Transition. J. Phys. Chem. B 2011, 115, 7736-7743.

(77) Capaccioli, S.; Ngai, K. L.; Ancherbak, S.; Paciaroni, A. Evidence of Coexistence of Change of Caged Dynamics at T-G and the Dynamic Transition at T-D in Solvated Proteins. J. Phys. Chem. B 2012, 116, 1745-1757.

(78) Hetzel, R.; Wuthrich, K.; Deisenhofer, J.; Huber, R. Dynamics of Aromatic Amino-Acid Residues in Globular Conformation of Basic
Pancreatic Trypsin-Inhibitor (Bpti) 0.2. Semiempirical Energy Calculations. Biophys. Struct. Mech. 1976, 2, 159-180.

(79) Gelin, B. R.; Karplus, M. Sidechain Torsional Potentials and Motion of Amino-Acids in Proteins - Bovine Pancreatic TrypsinInhibitor. Proc. Natl. Acad. Sci. U. S. A. 1975, 72, 2002-2006.

(80) Hong, L.; Cheng, X. L.; Glass, D. C.; Smith, J. C. Surface Hydration Amplifies Single-Well Protein Atom Diffusion Propagating into the Macromolecular Core. Phys. Rev. Lett. 2012, 108, 238102.1238102.4.

(81) Frauenfelder, H.; Chen, G.; Berendzen, J.; Fenimore, P. W.; Jansson, H.; McMahon, B. H.; Stroe, I. R.; Swenson, J.; Young, R. D. A Unified Model of Protein Dynamics. Proc. Natl. Acad. Sci. U. S. A. 2009, 106, 5129-5134. 